\title{
User Experience Evaluation of People-Dominant Service Interface
}

\author{
Ryota Mori (mori@nagano-kentan.ac.jp) \\ Nagano Prefectural College, 8-49-7, Miwa, Nagano City, 380-8525, Japan
}

\begin{abstract}
The role of the service interface has become more and more important for user to obtain their experience effectively and efficiently. With help of various service system, one can find a good experience. However, the past studies have not answered the question of the service interface. The purpose of my work was to e xamine which service interface is best to have an experience on the site. The purpose of this paper is to re port on my work concerning people-dominant interface design for services system. For the purpose of my w ork, two kinds of investigations were made on field using questionnaire and observation to compare expectat ion and user experience in several service systems. Studies have been made on which service interface is $b$ est. The study has clearly confirmed the fact that people-dominant interface with emotional connection indic ated the largest number of expected values in two restaurants and four shops. Moreover, mental model meas urement methods with kappa coefficient are used for comparison purposes. As a result, the theme park had a potential problem for user experience design. In conclusion, service interface with emotional interaction is the best system among a plurality of stores. Additionally, based on two theme park comparison results, it is recommended that the theme park be still improved in terms of task level.
\end{abstract}

Keywords: Service interface, User experience, Service design

\section{Introduction}

We conducted studies that measure user reaction to people-dominant service interface. Our purpose is to examine the effectiveness of their interface, and how an interaction with a waiter can prove powerful in exceeding user expectations.

In order to continuously provide a high-quality user experience, it is necessary to predict interactions with services and products, and to exceed user expectations. The organization were challenged to continue delivering a quality user experience.

The interface type of the service is divided into three types: people-dominant, machine-dominant, and hybrids of people and machines.

In this research, we focused on eating and drinking establishment and theme park as a system and explored the experience in people-dominant interface. As a quantification method, the experience is measured by questionnaire and UX curve.

Moreover, the theme park is used as a case example, and the purpose is to evaluate the service using the quantification method applicable to user interface evaluation and to examine the effectiveness.

\section{Method}

Two subjects were surveyed. The first one is a restaurant (C) in the Tokyo metropolitan area and a theme type restaurant $(\mathrm{K})$, and the participants are 5 female students. The second one is a bakery (A), a pottery shop (T), a cafe $(\mathrm{L})$, and a restaurant $(\mathrm{R})$ in Nagano prefecture, and the participants are 5 female students.

To the participants, questionnaire survey was conducted before and after, and the expected value was calculated. Also, they described the UX curve after it, and was digitized the vertical axis as VAS (Visual Analog Scale).

The subjects to be evaluated are two theme parks, here F and D. The participating group of F, 2 female students in $t$ a total of 5 . The participating group of $D$ is 
6 female students in total.

Participants created each experience curve (UX curve) after the incident. At the time of analysis, the vertical axis is converted into a numerical value as VAS (Visual Analog Scale).

We compared the behavioral results of the target group of $\mathrm{D}$ with the recommended plan to fully enjoy the attraction by experts and calculated the coincidence rate. In F, the coincidence rate between 2 and 5 people is calculated.

When asking for a response I got an agreement on the explanation with the document and do not present a reward.

At the time of requesting a response, we got an agreement of explanation orally with the document, we do not present a reward and no expenses such as entrance fee will be paid.

Participants did not plan detailed in advance and taken voluntary action in advance. The investigators did shadowing, accompanied the participating groups without active interaction.

\section{Results}

Both Tokyo and Nagano UX curve values (score / maximum value) are related to the posteriori.

\subsection{Service interface of the restaurant and shop}

The staying time in Tokyo was from noon to $19 \mathrm{pm}$. As $\mathrm{S}$, which was scheduled to enter at first, was changed to a different shop because it was full at the reservation, the preliminary evaluation value was not changed, and it was taken as the target store for ex-post evaluation. Next entered in $\mathrm{K}$.

$\mathrm{K}$ was a system for providing a service having an interface specialized for people compared with $\mathrm{S}$. K was decorated like a cave, and there was a grill in the private room of the audience seating, a feature that the store clerk who dressed as a monster threatened the customer and the unique dish suitable for the atmosphere was seen. In addition, $\mathrm{S}$ specializes in pizza with a reputation overseas.

For two service systems, high satisfaction was shown as a result of acquiring the prior and subsequent expected values and describing the UX curve.

The staying time in Nagano was from 10 am to $18 \mathrm{pm}$ and it went in the order of A, T, L, R. Depending on the circumstances of the time, we changed the original schedule, and $\mathrm{R}$ is visiting another shop.

$\mathrm{T}$ is not a restaurant but you can experience pottery and have a homey atmosphere. Compared to A, L and R, $\mathrm{T}$ has a specialized interface for people. Regarding the four service systems, as a result of obtaining advance and post expectation values and describing UX curves, overall high satisfaction was shown.

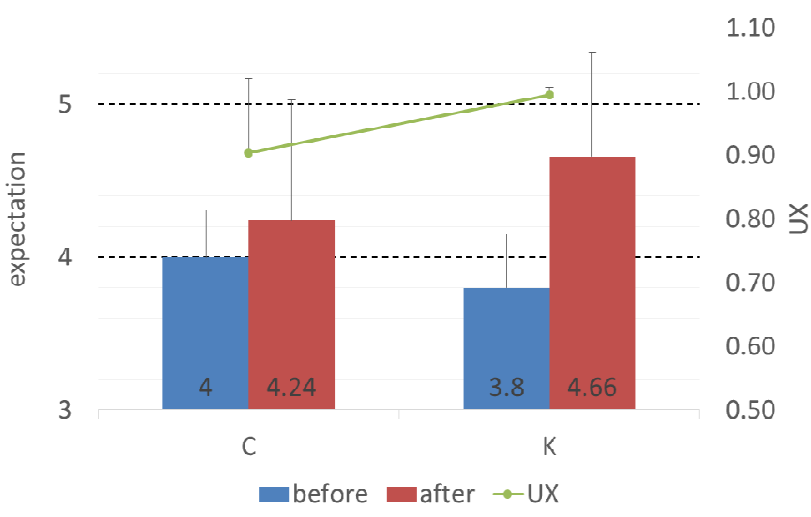

Figure 1. The expected values in the restaurant

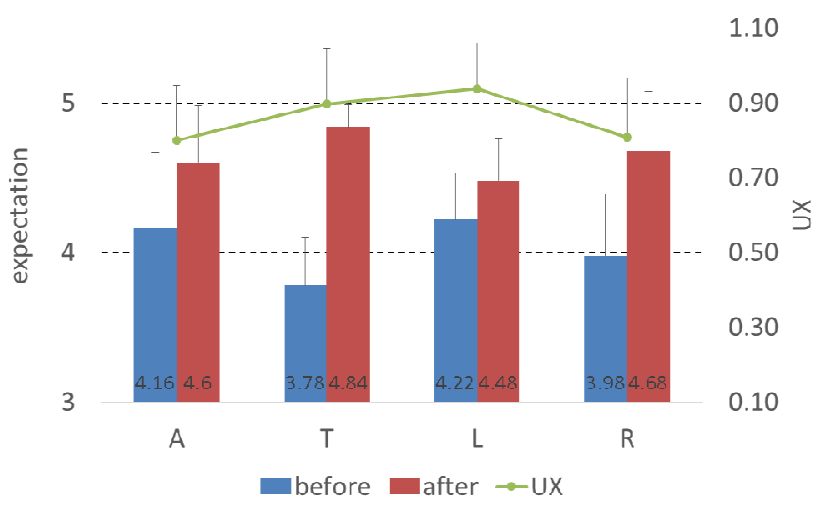

Figure 2. The expected values in the shop

\subsection{Service interface of the theme park}




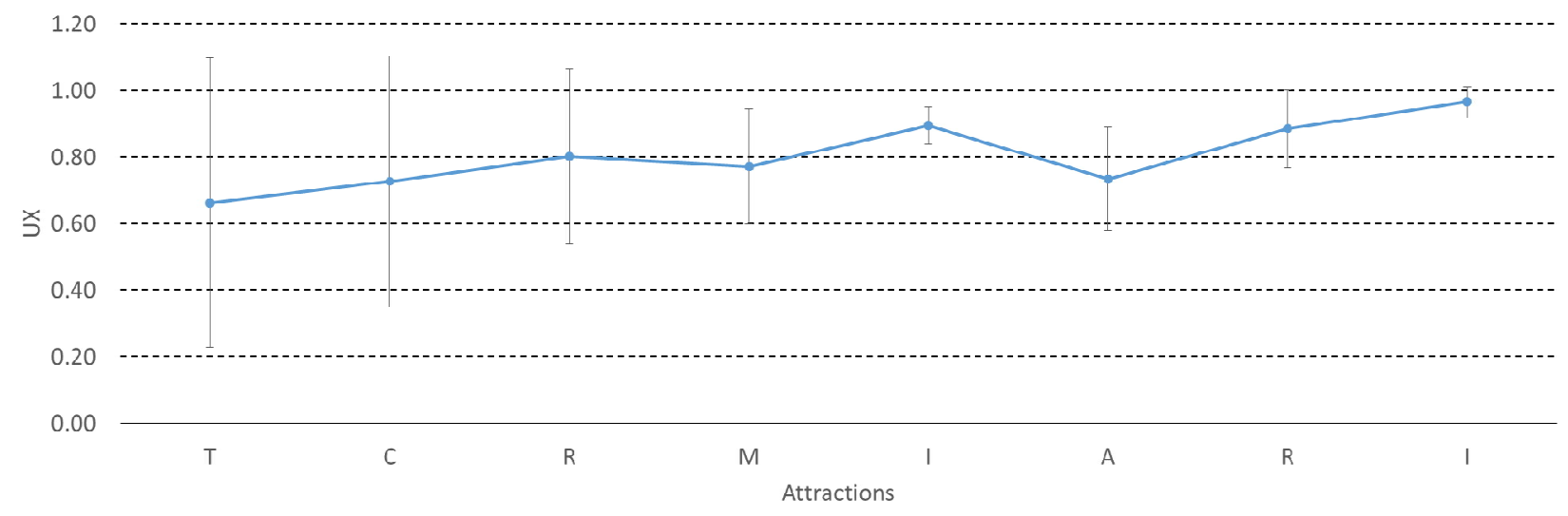

Figure 3. The UX values at each attraction

The main attraction of $\mathrm{D}$ was the action to be done in the morning, the coincidence rate was $\kappa=0.25$. In F, dis satisfaction with attraction was seen in the UX curve, sh owing $\kappa=0.21$. UX degree (score / maximum score) in each attraction was calculated from the UX curve. Fig.3 shows the variation in UX degree on attractions measured with VAS. The coincidence rate is lower tha $n$ the UX degree. The results obtained show that des pite their simplicity, these methods give relatively a ccurate results and are sufficient for user experience design evaluation.

\section{Discussion}

A highly experienced system was found in the system where ingenuity was found in the human specialized interface. As a factor that exceeded advance expectations, $\mathrm{K}$ has a human trick that can experience a fear experience, and $\mathrm{T}$ is considered to have led to good experiences in communication with shop clerks, in addition to manufacturing experience. We quantified that customer service communication becomes an important point to enhance UX.

$\mathrm{D}$, there is no similar tendency in the performance result and the subjective evaluation result from the concordance rate of expert route and participant route and measurement result by UX curve. Participants were able to finish the main attraction while the waiting time in the morning was little this time, so it seems that it was subjectively very satisfying feelings. Besides that, the factors of the difference in waiting time occurring between events will affect the discrepancy. In F, the disagreement between waiting time due to attraction and expectation is reflected.

Although the satisfaction level is indicated from the UX curve, the agreement between the result reflecting the functional model and the recommended plan is low. This may imply latent dissatisfaction of invisible users from subjective evaluation values. If they go according to the recommended plan, there is a possibility that it will be a higher quality experience.

Humans and machines are fused, and effective interaction is desired. On the other hand, it is requested that a person behaves like a machine, and the machine behaves like a person.

In order to improve the service, it is required to examine the possibility of user dissatisfaction and whether it has value suitable for the amount of money, predict interaction, and exceed the expected expectation.

However, it is difficult only by subjective assessment with a questionnaire to see frustration firmly.

The study showed the people-dominant interface to be more effect, that is, users had it more than that they needed to get a satisfaction

Meanwhile, in the usability testing of products, methods based on comparison with experts are utilized and the effectiveness is shown, but there are few cases applied to evaluation of services. The disagreement between experts and users remain as one of the key is sues to be clarified.

As a future application plan, evaluation using a persona can be considered. Using a persona mental model as a criterion, it will be possible to evaluate whether or not the service designer has obtained the experience as intended by the user based on the coincidence rate of this method.

\section{Conclusion}


In order to consider better service, it is necessary not only to individual interfaces, but also to look over the w hole interface as a bird's-eye view.

From the comparison of interface and interface, it can be concluded that people dominant interface is effective.

The results show that service improvement is pos sible using people-dominant interface suggest that th is can be achieved using standard emotional connec tions.

\section{References}

Tom Tullis, Bill Albert, Measuring the User Experience, Collecting, Analyzing, and Presenting Usability Metrics, Elsevier, 2008

John A. Goodman, Customer Experience 3.0: High - Profit Strategies in the Age of Techno Service., AMACOM, 2014.

Jeffrey F. Rayport, Bernard J. Jaworski : Best Face Forward: Why Companies Must Improve Their Service Interfaces With Customers, 2004

Cohen, Jacob (1960). "A coefficient of agreement for nominal scales". Educational and Psychological Measurement 20 (1): pp.37-46.

Ryota Mori, A Proposal for Measurement Methods of Mental Models Using Kappa Coefficient, Japan Society of Kansei Engineering, Vol. 15 (2016) No. 4 